\title{
LHomme
}

L'HOMME Revue française d'anthropologie

$190 \mid 2009$

Varia

\section{Henrika Kuklick, ed., A New History of Anthropology}

\section{Nélia Dias}

\section{OpenEdition}

\section{Journals}

Édition électronique

URL : http://journals.openedition.org/lhomme/28716

DOI : 10.4000//homme.28716

ISSN : 1953-8103

\section{Éditeur}

Éditions de l'EHESS

\section{Édition imprimée}

Date de publication : 1 janvier 2009

Pagination : 208-210

ISSN : 0439-4216

\section{Référence électronique}

Nélia Dias, "Henrika Kuklick, ed., A New History of Anthropology », L'Homme [En ligne], 190 | 2009, mis en ligne le 03 janvier 2017, consulté le 24 septembre 2020. URL : http://journals.openedition.org/ Ihomme/28716 ; DOI : https://doi.org/10.4000//homme.28716

Ce document a été généré automatiquement le 24 septembre 2020.

(c) École des hautes études en sciences sociales 


\title{
Henrika Kuklick, ed., A New History of Anthropology
}

\author{
Nélia Dias
}

\section{RÉFÉRENCE}

Henrika Kuklick, ed., A New History of Anthropology, Oxford-Malden, Blackwell, 2008, 402 p., bibl., index, ill.

COMME L'INDIQUE le titre de cet ouvrage, c'est à une véritable nouvelle histoire de l'anthropologie que nous convie Henrika Kuklick, et ce pour trois raisons. Premièrement, par son angle d'approche, A New History of Anthropology cherche à rendre compte aussi bien des diverses traditions nationales que des concepts, des grilles d'analyse, des domaines de recherche et des sous-disciplines en anthropologie. De ce point de vue, cet ouvrage rompt avec les histoires «classiques» de l'anthropologie ordonnées selon un découpage chronologique et centrées sur les pères fondateurs ou les "progrès» des théories anthropologiques. Deuxièmement, par le dialogue constamment entretenu entre le passé du savoir anthropologique et le présent, ce livre vise moins à mettre en lumière des filiations méconnues et des figures oubliées, qu'à accentuer, comme le souligne Henrika Kuklick, «significant differences between past and present practitioners» (p.7). D'ailleurs, le plan du livre comprenant dix-neuf articles organisés en cinq sections - «Les traditions majeures", "Anciennes obsessions», "Les passés négligés», «Biologie» et "Nouvelles directions et perspectives »- atteste de cette volonté de confronter les différences, à la fois thématiques et méthodologiques, qui séparent l'anthropologie du xix siècle de celle de nos jours. Troisièmement, par son dessein d'« apporter une perspective historique aux débats contemporains ", cet ouvrage invite à repenser un certain nombre de questions centrales à ce domaine de connaissance telles que ses liens controversés avec le colonialisme, ses frontières avec d'autres champs disciplinaires, la distinction 
problématique entre anthropologie académique et anthropologie appliquée et les rapports entre la sphère biologique et la sphère culturelle.

Dans son introduction solide et bien argumentée, Henrika Kuklick explicite les thèmes majeurs qui traversent ce livre. D'une part, elle met en relief la prégnance du paradigme de l'« anthropological salvage » et la façon dont l'impératif de l'extinction culturelle, présidant à la colonisation, reste encore puissant à l'heure de la mondialisation. Les remarques nuancées de l'auteure pour ce qui est des "pêchés originaux » de l'anthropologie, à savoir ses liens complexes avec le colonialisme, sont étayées par les contributions d'Anna Grimshaw et de Donna Mehos, la première consacrée à l'essor de l'anthropologie visuelle, la seconde aux musées ethnographiques aux Pays-Bas. D'autre part, Henrika Kuklick examine la question (toujours ouverte) des frontières de l'anthropologie dans le sillage des analyses de George W. Stocking ${ }^{1}$. Elle note pertinemment à quel point le souci de "pureté disciplinaire " tend à proliférer pendant les « périodes de pénurie de ressources économiques » (p. 3) allant de pair avec la réduction du nombre de postes dans les institutions d'enseignement supérieur. En associant étroitement le problème de la délimitation des frontières en anthropologie au marché du travail, Henrika Kuklick déplace les termes du débat pour le situer sur le terrain des débouchés professionnels ; et l'auteure de rappeler, à juste titre, les travaux de Ruth Benedict, de Franz Boas et de Margaret Mead à la charnière de la science " pure » et de la science « appliquée».

Deux contributions dans ce volume témoignent, par des voies tout à fait distinctes, de la part des enjeux institutionnels et professionnels dans les orientations théoriques de l'anthropologie. Robert Ackerman, par ailleurs auteur de J. G. Frazer, his Life and Work', retrace, avec talent et érudition, les rapports qu'a entretenus l'anthropologie avec l'étude des sociétés antiques. Si, à la fin du XIXe siècle, l'étude des sociétés antiques, en raison de leur statut élevé dans le champ académique, a pu constituer un modèle de référence pour le savoir émergent qu'était l'anthropologie, il en est tout autrement dans les années 1960 ; avec la progressive marginalisation des études classiques au sein de l'université, ce sont les méthodes et les théories anthropologiques qui vont nourrir les travaux des classicisants et contribuer fortement au développement d'une anthropologie des sociétés antiques. Merrill Singer, quant à lui, s'attache à l'émergence et à la consolidation de l'anthropologie appliquée aux États-Unis, notamment au sein de l'American Anthropological Association (AAA). Deux dates jalonnent ce mouvement: 1941, la création de la Society for Applied Anthropology avec le concours, entre autres, de Margaret Mead et de Gregory Bateson et 1983, la fondation de la National Association for Practicing Anthropologists (NAPA). Le développement, à la fin des années 1970, d'une "Nouvelle anthropologie appliquée», centrée sur la santé, l'environnement, l'éducation et les droits de l'homme, n'est pas sans rapports, selon l'auteur, avec, d'un côté, l'effondrement du marché du travail au sein de l'université, et avec, de l'autre, l'essor de nouveaux débouchés professionnels dans le cadre d'organismes gouvernementaux et non gouvernementaux. Et Merrill Singer de soutenir que l'avenir de l'anthropologie passe par la dimension appliquée : «practice is not an option for anthropology if it is to survive; it is the discipline's destiny » (p. 327). Pour polémique que soit cette assertion, elle a le mérite d'inviter les anthropologues à réfléchir sur la destinée de leur discipline ${ }^{3}$.

Il ne saurait être question, dans ce cadre limité, de rendre compte de façon exhaustive de la totalité des articles contenus dans ce riche volume. La section «Les traditions 
majeures" contient des synthèses denses et bien documentées, portant sur l'anthropologie nord-américaine (Regna Darnell), britannique (Henrika Kuklick) et dans les pays de langue allemande (H. Glenn Penny); Emmanuelle Sibeud, pour sa part, examine "Les métamorphoses de l'ethnologie en France, 1839-1930 ", en mettant en relief le rôle des administrateurs coloniaux dans les anciennes colonies françaises en Afrique. La section « Anciennes obsessions » inclut, outre l'article de Robert Ackerman, deux autres textes, l'un sur l'approche anthropologique de la religion (Ivan Strenski), l'autre sur la perception et la terminologie des couleurs (Barbara Saunders). Comme son titre le laisse entrevoir, la section « Les passés négligés » s'attache à l'anthropologie dans les pays "périphériques ", tels que les pays nordiques avec l'analyse des écoles de Westermarck et de Nordenskiöld (Christer Lindberg) et la Russie à la fin du XIX siècle (Nikolai Ssorin-Chaikov). Avec des essais consacrés à la réception du darwinisme (Thomas F. Glick), à l'influence de la notion de race dans la division entre anthropologie physique et anthropologie culturelle aux États-Unis (Jonathan Marks) et aux critères présidant à la séparation entre le règne humain et le monde animal (Robert N. Proctor), A New History of Anthropology rend justice à la dimension biologique, très souvent négligée dans les histoires de la discipline.

5 La dernière section, "Nouvelles directions et perspectives", s'attelle aussi bien aux sous-domaines en anthropologie (l'anthropologie visuelle et l'anthropologie appliquée) qu'aux reconfigurations de la notion de terrain. C'est l'un des mérites de l'article de Lyn Schumaker de s'attarder sur la façon dont les femmes, par leur travail de terrain, ont influencé les orientations de la discipline anthropologique. En distinguant soigneusement l'histoire des femmes en anthropologie de l'histoire du genre et de la sexualité en tant que domaines de recherches, elle propose d'examiner le rôle qu'ont joué les femmes pour ce qui est du travail de terrain, à l'instar d'autres figures, telles que les missionnaires et les administrateurs. Qu'en est-il de la notion d'aire culturelle, en tant qu'outil d'analyse en anthropologie, à l'heure de la mondialisation? Telle est la question magistralement posée par Rena Lederman dans son article « Anthropological Regionalism ». On sait à quel point la définition d'aires culturelles, à partir de critères géographiques, écologiques, linguistiques ou d'un ensemble d'éléments culturels, a pu compter dans l'élaboration des théories anthropologiques. Or, ce qui était l'apanage du savoir anthropologique, à savoir l'expérience de terrain géographiquement délimité, s'effrite graduellement, selon Rena Lederman, du fait du questionnement des dichotomies local/global, dedans/dehors et de la prise en considération d'un terrain devenu de plus en plus multisitué et multilocal.

6 Éditrice de History of Anthropology Newsletter, Henrika Kuklick est bien placée pour présenter des histoires plutôt qu'une histoire canonique de l'anthropologie. Son livre intervient après plusieurs autres ${ }^{4}$, mais il se démarque par sa volonté de ne pas fournir une histoire encyclopédique, par la liberté laissée au lecteur de ne pas suivre forcément l'ordre des chapitres et par son approche riche en perspectives pour aujourd'hui. 


\section{NOTES}

1. Voir George W. Stocking Jr., "Delimiting Anthropology: Historical Reflections on the Boundaries of a Boundless Discipline", in Delimiting Anthropology. Occasional Inquiries and Reflections, Madison, University of Wisconsin Press, 2001 : 303-329.

2. Cambridge-New York, Cambridge University Press, 1987.

3. Voir à ce sujet, les remarques pertinentes de Maurice Bloch, « Where Did Anthropology Go ? Or the Need for "Human Nature" ", in Essays on Cultural Transmission, Oxford-New York, Berg, 2005 : 1-19.

4. C'est dans cette perspective que s'inscrivent les ouvrages de Fredrik Barth et al., One Discipline, Four Ways. British, German, French, and American Anthropology, Chicago, University of Chicago Press, 2005 et celui de Thomas Hyllans Eriksen \& Finn Sivert Nielsen, A History of Anthropology, London, Pluto Press, 2001.

\section{AUTEURS}

\section{NÉLIA DIAS}

Université de Lisbonne, Lisbonne (Portugal).

nelia-dias@clix.pt 